\title{
ANÁLISIS DE LA RELACIÓN DE LA INNOVACIÓN EMPRESARIAL CON LA FINANCIACIÓN EN COLOMBIA*
}

\author{
Bernardo Barona Zuluaga** \\ Jorge Alberto Rivera Godoy ${ }^{* * *}$ \\ Carlos Iván Aguilera Cifuentes ${ }^{* * * * * *}$
}

* doi: doi:10.11144/Javeriana.cao28-50.arie. Este artículo es producto de la investigación “Proyecto CI-8104: La financiación de la innovación en Colombia" financiado por la Universidad del Valle desde el 11 septiembre de 2012 hasta el 11 de julio de 2015. El artículo se recibió el 14/11/2014 y se aprobó el 30/05/2015. Sugerencia de citación: Barona Z., B., Rivera G., J. A. y Aguilera C., C. I. (2015). Análisis de la relación de la innovación empresarial con la financiación en Colombia. Cuadernos de Administración, 28 (50), 11-37. http://dx.doi. org/10.11144/Javeriana.cao28-50.arie

** Doctor of Philosophy, Universidad de Manchester, Inglaterra, 1985. Profesor pensionado del Departamento de contabilidad y finanzas de la Universidad del Valle, Cali, Colombia.

Correo electrónico: bbaronaz@gmail.com

*** Doctorado en Ciencias Económicas y Empresariales. Con calificación de sobresaliente Cum Laude de la Universidad Autónoma de Madrid, España, 1999. Docente nombrado tiempo completo del Departamento de contabilidad y finanzas de la Universidad del Valle, Cali, Colombia.

Correo electrónico:jorge.rivera@correounivalle.edu.co

**** Doctorado en Administración de Empresas de FGV de Sao Paulo, Brasil, 1998. Profesor del Departamento de administración y organizaciones de la Universidad del Valle, Cali, Colombia.

Correo electrónico: carlos.aguilera@correounivalle.edu.co 


\section{Análisis de la relación de la innovación empresarial con la financiación en Colombia}

\section{RESUMEN}

Este artículo examina si las empresas innovadoras tienen un patrón de financiación diferente al de las empresas no innovadoras, y analiza la relación entre actividades de innovación y fuentes de financiación a la luz de la teoría económica. Se realizan test estadísticos con base en la información de la Encuesta Empresarial del Banco Mundial de 2010 y se encuentra que las empresas innovadoras utilizan diferentes fuentes de financiación (recursos internos, financiación bancaria y crédito de proveedores para financiar capital de trabajo) al que hacen las empresas no innovadoras. Las principales fuentes de financiación de las empresas innovadoras son los recursos propios y la deuda bancaria. Para futuras investigaciones se sugiere profundizar estos hallazgos con encuestas y estudios de caso.

Palabras clave: financiación de la innovación, empresas innovadoras, finanzas de emprendedores.

Clasificación JEL: $\mathrm{G}_{32}, \mathrm{M}_{13}$

Analysis of the relation between entrepreneurial innovation and finance in Colombia

\section{ABSTRACT}

This study examines whether innovative companies have a different financing pattern compared to that of non-innovative enterprises, and it analyzes the relation between innovation activities and specific sources of funding within the scope of economic theory. Statistical tests conducted using the World Bank Enterprise Survey 2010 as a source suggest that innovative companies make use of certain sources of funding (internal resources, bank financing and credit from suppliers to finance working capital) that are absent in non-innovative enterprises. Moreover, the main financing sources of innovative companies are own resources and bank debt. Further surveys and studies are suggested to deepen the reported findings.

Keywords: Financing innovation, innovative companies, financing for entrepreneurs.

JEL Classification: $\mathrm{G}_{32}, \mathrm{M}_{13}$

\section{Análise da relação da inovação empresarial com o financiamento na \\ Colômbia}

\section{Resumo}

Este artigo examina se as empresas inovadoras têm um padrão de financiamento diferente ao das empresas não inovadoras e analisa a relação entre atividades de inovação e fontes de financiamento à luz da teoria econômica. Realizam-se testes estatísticos com base na informação do World Bank Enterprise Survey Data de 2010 e constata-se que as empresas inovadoras fazem um uso diferente das fontes de financiamento (recursos internos, financiamento bancário e crédito de fornecedores no financiamento do capital de trabalho) ao que as empresas não inovadoras fazem. As principais fontes de financiamento das empresas inovadoras são os recursos próprios e a dívida bancária. Sugere-se aprofundar essas constatações com pesquisas e estudos de caso.

Palavras-chave: financiamento da inovação, empresas inovadoras, finanças de empreendedores.

Classificação JEL: G32, M13. 


\section{Introducción}

El propósito de este estudio es contribuir al conocimiento que se tiene en Colombia de la manera en que las empresas privadas financian actividades de innovación y de investigación y desarrollo (I+D). La fuente de información que se emplea es el Enterprise Survey del Banco Mundial (WBES) correspondiente al año 2010. Con base en las respuestas dadas por directivos de las 942 empresas incluidas en el Survey a preguntas sobre las fuentes de financiación utilizadas y actividades de innovación y de I+D llevadas a cabo, el estudio busca identificar si las empresas innovadoras exhiben patrones de financiación diferentes de los de las no innovadoras, y analizar las asociaciones existentes entre ser, 0 no, innovadora y haber invertido en $I+D$, utilizando fuentes de financiación específicas. Adicionalmente, se busca reconocer la asociación entre la realización de actividades de innovación y de I+D con características empresariales, como tener cuenta bancaria, o contar con inversión extranjera, así como con la percepción de los encuestados respecto a si la financiación ha sido un obstáculo para el desarrollo de la empresa. El tema de la estructura óptima de capital no es examinado aquí debido al interés fundamental de comprender las relaciones innovación vs. fuentes de financiación, y porque las preguntas en el survey hacían referencia a la financiación de inversiones especificas en el capital de trabajo o en los activos fijos, y no a la financiación de la empresa en su conjunto.

Este artículo se ha estructurado en cinco secciones: en la primera se presentan los principales desarrollos teóricos y empíricos sobre la financiación en empresas innovadoras, como también las principales características de las empresas innovadoras en Colombia; en la segunda se expone el método empleado para la recolección y análisis de la información; en la tercera se presentan los principales hallazgos; en la cuarta se analizan los resultados y se contrastan con desarrollos teóricos y evidencias empíricas; finalmente, en la quinta sección se presentan las conclusiones.

\section{Marco de referencia}

En el marco de la teoría económica neoclásica se han identificado varias razones por las cuales las empresas que operan en mercados completamente libres tienden a realizar menos inversión en investigación, desarrollo e innovación de la considerada óptima para alcanzar altos niveles de crecimiento y desarrollo económico (Arrow, 1962; Hall, 2002; Hall y Lerner, 2010). Una razón importante para esto es que un porcentaje muy elevado de esta inversión está representada en remuneración a personal altamente calificado, como científicos e ingenieros. Aunque conceptualmente estos pagos constituyen una in- 
versión para la empresa, por su naturaleza intangible es una inversión muy diferente a la inversión en activos físicos como inventario en mercancías o planta y equipo (Hall, 2002; Hall y Lerner, 2010). Quien va a financiar esta inversión es consciente de esta naturaleza y de que los activos, además de ser intangibles, no se pueden considerar como propiedad de la empresa de la misma manera que los activos físicos, por tanto si las personas dejan la empresa, ésta pierde la inversión (Rajan y Zingales, 2003). Otro factor diferenciador de la inversión en $I+D$ es que existe un grado de incertidumbre mayor en relación con los resultados de esta que el existente con otro tipo de inversiones ${ }^{1}$. Esta incertidumbre tiende a ser muy alta al comienzo del proyecto, lo que implica que los métodos tradicionales de evaluación de inversiones con frecuencia no resultan apropiados en estos casos. Una alternativa a los proyectos de investigación y desarrollo ejecutados por las empresas es recurrir al conocimiento y recursos existentes en el entorno, aplicando estrategias de innovación abierta.

La teoría de los costos de transacción resalta en su análisis de la relación inversión - financiación las peculiaridades de los activos que resultan de la inversión y los contratos de financiación más apropiados para financiar esta inversión (Williamson, 1988). Al contrastar las características de la inversión en activos intangibles (típica de la innovación en empresas del sector de las Tecnologías de la Información y las Comunicaciones: TIC) con la inversión en activos tangibles, Williamson (1996) concluye que los costos de la financiación con deuda son más altos que los de la financiación con patrimonio. Hall y Lerner (2010) encuentran que la financiación de la inversión en I+D en países desarrollados respalda las conclusiones de la mayor conveniencia de los recursos internos y de las ventajas de la financiación mediante emisión de acciones en relación con la financiación de deuda. No obstante, en países emergentes que se caracterizan, entre otras cosas, por carecer de mercados de acciones desarrollados, varios estudios han encontrado que la disponibilidad de financiación bancaria es un importante determinante de la actividad emprendedora empresarial (Ayyagari, Demirgüc-Kunt y Maksimovic, 2011). Otras investigaciones que han explorado las diferentes facetas de la financiación de la innovación son Hall (2002), Carpenter y Petersen (2002), Nanda y Rhodes-Kropf (2013a, 2013b) y Kerr y Nanda (2014). Aunque en Colombia la literatura en que se relaciona la actividad innovadora con su financiación es escasa ha permitido identificar algunos aspectos de

1 Sierra, Malaver y Vargas (2009) analizan la incertidumbre en relación con la innovación y discuten en detalle conceptos desarrollados tanto por 0'Sullivan (2005) como por Léger y Swaminathan (2007); además identifican la necesidad de la intervención del Estado y de políticas de ciencia, tecnología e innovación que estimulen las inversiones del sistema empresarial. 
interés tanto para la empresa como para la política pública, incluyendo la importancia de la financiación proveniente de los recursos internos/utilidades retenidas y de la banca como fuente de recursos para la innovación; un hecho interesante, desde el punto de vista organizacional y de política pública, es la existencia de una relación positiva entre el tamaño de las empresas y la realización de actividades innovadoras (Langebaek y Vásquez, 2007; Sierra et al., 2009; Arbeláez y Parra, 2011; García, Barona y Madrid, 2013; Rivera y Ruiz, 2009).

La investigación cuyos resultados se reportan aquí busca contribuir a la comprensión que se tiene sobre la relación innovación vs. fuentes de financiación en el ámbito empresarial en Colombia; se examina si las empresas innovadoras exhiben patrones de financiación diferentes de las no innovadoras y se inspeccionan algunas posibles relaciones entre el tipo de innovación y el uso de fuentes específicas de financiación, como las utilidades retenidas y la deuda bancaria. Utilizando información disponible en la base de datos WBES del Banco Mundial se analiza también la existencia de posibles relaciones entre el tipo de innovación realizada por las empresas y la variables edad y estatus legal, así como con las respuestas dadas a las preguntas acerca de si la empresa tiene cuenta bancaria y si ve la financiación como un obstáculo para la innovación.

La base de datos en la que se fundamenta este estudio es la WBES correspondiente al año 2010, la cual contiene información sobre 942 empresas colombianas. En la Tabla 1 se presentan las respuestas dadas a la pregunta de si las empresas hacen o no innovación y los principales tipos de innovación que realizan. También se muestran las respuestas a la pregunta de si tienen patentes en Colombia o en el exterior, y las respuestas a otras preguntas relacionadas con las actividades de innovación.

A continuación se resumen los aspectos más relevantes de la Tabla 1.

- Frecuencia de los diferentes tipos de innovación: el tipo de innovación más frecuente resultó ser: "procesos nuevos/significativamente mejorados para producir/suministrar productos", con aproximadamente el $43 \%$ de los encuestados indicando que realizan este tipo de innovación. Le siguen en orden de frecuencia: “productos nuevos/significativamente mejorados al mercado del establecimiento", con el $24,6 \%$ de respuestas positivas y, finalmente: "procesos nuevos/significativamente mejorados también nuevos para su industria", con el $22,6 \%$ de las respuestas positivas. 


\section{Tabla 1}

Características de la innovación realizada por las empresas colombianas

\begin{tabular}{|c|c|c|c|c|c|c|c|}
\hline \multirow{2}{*}{ Preguntas de interés para estudio de innovación } & \multicolumn{2}{|c|}{$\begin{array}{c}\text { No sabe / en } \\
\text { blanco }\end{array}$} & \multicolumn{2}{|c|}{ No } & \multicolumn{2}{|c|}{ Sí } & \multirow{2}{*}{$\begin{array}{l}\text { Total } \\
\text { No. } \\
\text { Emp. }\end{array}$} \\
\hline & $\begin{array}{l}\text { No. } \\
\text { Emp. }\end{array}$ & $\%$ & $\begin{array}{l}\text { No. } \\
\text { Emp. }\end{array}$ & $\%$ & $\begin{array}{l}\text { No. } \\
\text { Emp. }\end{array}$ & $\%$ & \\
\hline $\begin{array}{l}\text { DA. Últimos } 3 \text { años: ¿productos nuevos/significati- } \\
\text { vamente mejorados al mercado del establecimiento? }\end{array}$ & 510 & 54,1 & 200 & 21,2 & 232 & 24,6 & 942 \\
\hline $\begin{array}{l}\text { DB. Últimos } 3 \text { años: ¿procesos nuevos/significa- } \\
\text { tivamente mejorados para producir/suministrar } \\
\text { productos? }\end{array}$ & 238 & 25,3 & 299 & 31,7 & 405 & 43,0 & 942 \\
\hline $\begin{array}{l}\text { DC. Últimos } 3 \text { años: ¿procesos nuevos/significativa- } \\
\text { mente mejorados también nuevos para su industria? }\end{array}$ & 541 & 57,4 & 188 & 20,0 & 213 & 22,6 & 942 \\
\hline $\begin{array}{l}\text { DD. ¿ ¿Hizo el establecimiento desembolsos para acti- } \\
\text { vidades de I+D internas? }\end{array}$ & - & - & 301 & 32,0 & 404 & 42,9 & 942 \\
\hline $\begin{array}{l}\text { DF. Últimos } 3 \text { años: ¿coopera en innovación con otras } \\
\text { empresas /instituciones de ciencia y tecnología? }\end{array}$ & 238 & 25,3 & 542 & 58,0 & 162 & 17,2 & 942 \\
\hline $\begin{array}{l}\text { DG. Últimos } 3 \text { años: ¿recibe algún apoyo para activi- } \\
\text { dades relacionadas con innovación? }\end{array}$ & - & - & 605 & 64,23 & 100 & 10,62 & 942 \\
\hline $\begin{array}{l}\text { DJ. ¿Usa usted tecnología licenciada de una compa- } \\
\text { ñía extranjera? }\end{array}$ & 240 & 25,48 & 617 & 65,50 & 85 & 9,02 & 942 \\
\hline $\begin{array}{l}\text { DK ¿Tiene este establecimiento alguna patente en } \\
\text { el exterior? }\end{array}$ & 242 & 25,69 & 644 & 68,36 & 56 & 5,95 & 942 \\
\hline $\begin{array}{l}\text { DL ¿Tiene el establecimiento alguna patente regis- } \\
\text { trada? }\end{array}$ & 242 & 25,69 & 490 & 52,01 & 210 & 22,30 & 942 \\
\hline $\begin{array}{l}\text { DM ¿Gasta el establecimiento en compra de licencias } \\
\text { para usar propiedad intelectual? }\end{array}$ & 240 & 25,48 & 478 & 50,74 & 224 & 23,78 & 942 \\
\hline $\begin{array}{l}\text { D0. Últimos } 3 \text { años: ¿llenó aplicación para registro } \\
\text { de patentes/marcas comerciales/ derechos de autor } \\
\text { para productos/innovaciones de procesos? }\end{array}$ & 242 & 25,69 & 505 & 53,61 & 195 & 20,70 & 942 \\
\hline
\end{tabular}

Nota: DA a D0 son las abreviaturas que se usan en dicho Survey para las preguntas.

Fuente: elaboración propia, con base en Enterprise Survey-Raw Data (2010).

- Realización de desembolsos para investigación y desarrollo: el 42,9\% respondió haber adelantado este tipo de desembolsos. El valor promedio de la inversión de las empresas que sí adelantaron I+D fue de \$399 millones (calculado sobre 374 empresas que reportaron el valor).

- Otras características de la actividad innovadora: con respecto al grado de cooperación con otras organizaciones en la realización de actividades de innovación, las respuestas afirmativas fueron el 17,2\%, indicando que aproximadamente 1 de cada 6 empresas que hace innovación la realiza en cooperación con otras orga- 
nizaciones. El 10,6\% manifestaron haber recibido en los últimos tres años algún apoyo para actividades relacionadas con innovación. Un porcentaje ligeramente inferior ( $9 \%$ ) respondió afirmativamente a la pregunta de si usa tecnología licenciada a compañía extranjera. El 23,8\% respondió hacer inversiones en compras de licencias para usar propiedad intelectual. El 20,7\% respondió haber llenado aplicación para registro de patentes/marcas comerciales/derechos de autor / innovaciones de procesos.

\section{Método de recolección y análisis de la información}

La muestra inicial con las respuestas de 942 empresas fue depurada a 869 con el fin de tener información uniforme para el análisis financiero².

En la Tabla 2 se presentan las características de esta muestra en lo correspondiente a tamaño, organización jurídica y sector en el que opera la empresa. Igualmente se expone una desagregación del total de empresas en las categorías de innovadoras y no innovadoras. Se clasificaron como innovadoras aquellas empresas que respondieron afirmativamente a alguna de las preguntas DA, DB, DC, DD y DL de la Tabla 1 y que tenían además la razón Gastos I+D / Ventas mayor que cero. Los investigadores consideran importante resaltar lo siguiente: i) las empresas innovadoras tienen un promedio de edad mayor al de las no innovadoras, en aproximadamente 3,9 años; ii) la muestra no incluye empresas micro ni de creación reciente y parece tener una edad promedio superior a la edad promedio de las empresas Pymes y grandes colombianas ${ }^{3}$; iii) las empresas pequeñas, medianas y grandes estuvieron representadas casi por partes iguales en la muestra, con una ponderación un poco menor en las pequeñas y un poco mayor en las medianas; iv) la mayoría de las empresas tenían una estructura jurídica en la que la responsabilidad de los dueños era limitada; solo $20(2,3 \%)$ estaban inscritas en el mercado público de valores; v) en la muestra predominan las empresas del sector manufacturero $(74,2 \%)$. Solo empresas de este sector indicaron hacer algún tipo de innovación.

2 Se eliminaron los registros de empresas cuya razón "Total Gastos de I+D /Ventas anuales" no se pudo calcular porque los encuestados indicaron no saber si habían realizado gastos de $\mathrm{I}+\mathrm{D}$, o no conocer el valor de las ventas anuales.

3 Conforme a información divulgada en septiembre de 2013 por la Confederación Colombiana de Cámaras de Comercio (Confecámaras), la cual es tomada del Registro Único Empresarial y Social (RUES) que lleva dicha institución, la vida promedio de las grandes compañías es de 18 años, mientras que para las Pymes es de 12 años (Lozano, 2013). 


\section{Tabla 2}

Características de las empresas de la muestra (base de datos depurada)

\begin{tabular}{|c|c|c|c|c|c|c|}
\hline$N=869$ & \multicolumn{2}{|c|}{ No innovadoras } & \multicolumn{2}{|c|}{ Innovadoras } & \multicolumn{2}{|c|}{ Total empresas } \\
\hline Número empresas & \multicolumn{2}{|c|}{589,0} & \multicolumn{2}{|c|}{280,0} & \multicolumn{2}{|c|}{869,0} \\
\hline Porcentaje & \multicolumn{2}{|c|}{67,8} & \multicolumn{2}{|c|}{32,2} & \multicolumn{2}{|c|}{100,0} \\
\hline \multirow[t]{2}{*}{ Promedio de edad años } & \multicolumn{2}{|c|}{22,2} & \multicolumn{2}{|c|}{26,1} & \multicolumn{2}{|c|}{23,5} \\
\hline & No. Emp. & $\%$ & No. Emp. & $\%$ & No. Emp. & $\%$ \\
\hline \multicolumn{7}{|l|}{ Tamaño } \\
\hline Pequeña $>=5 y<=19$ & 185 & 31,4 & 71 & 25,4 & 256 & 29,5 \\
\hline Mediana $>=20 \quad y<=99$ & 223 & 37,9 & 101 & 36,1 & 324 & 37,3 \\
\hline Grande $>=100$ & 181 & 30,7 & 108 & 38,6 & 289 & 33,3 \\
\hline Total & 589 & 100,0 & 280 & 100,0 & 869 & 100,0 \\
\hline \multicolumn{7}{|l|}{ Organización jurídica } \\
\hline Sociedad colectiva limitada & 297 & 50,4 & 107 & 38,2 & 404 & 46,5 \\
\hline Sociedad colectiva general & 5 & 0,9 & 2 & 0,7 & 7 & 0,8 \\
\hline Cía. de responsabilidad Ltda. de naturaleza privada & 205 & 34,8 & 134 & 47,9 & 339 & 39,0 \\
\hline Cía. listada en mercado público de valores & 12 & 2,0 & 8 & 2,9 & 20 & 2,3 \\
\hline Empresa unipersonal & 31 & 5,3 & 16 & 5,7 & 47 & 5,4 \\
\hline Otras & 39 & 6,6 & 13 & 4,6 & 52 & 6,0 \\
\hline Total & 589 & 100 & 280 & 100,0 & 869 & 100,0 \\
\hline \multicolumn{7}{|l|}{ Sector } \\
\hline Core & 115 & 19,5 & - & - & 115 & 13,2 \\
\hline Manufactura & 365 & 62,0 & 280 & 100 & 645 & 74,2 \\
\hline Comercio al detal & 109 & 18,5 & - & - & 109 & 12,5 \\
\hline Total & 589 & 100,0 & 280 & 100 & 869 & 100,0 \\
\hline
\end{tabular}

Fuente: elaboración propia, con base en Enterprise Survey-Raw Data (2010).

\subsection{Modelos para el análisis de variables}

Primero se realizaron varias pruebas de hipótesis donde la hipótesis principal es que las organizaciones utilizan fuentes de financiación diferentes, dependiendo de si son, o no, innovadoras.

Para someter a prueba esta hipótesis general se formularon las siguientes hipótesis específicas para cada una de las razones financieras (Yi) relacionadas con la financiación 
del capital de trabajo y con los activos fijos. La prueba estadística que se hizo es la denominada Mann- Whitney (MW) la cual determina si las dos poblaciones son idénticas4:

Ho: las dos poblaciones son idénticas.

Ha: las dos poblaciones no son idénticas.

La variable Yi (razón financiera) puede asumir cinco valores: 1 para el porcentaje de capital de trabajo (C de T) financiado por medio de fondos internos / utilidades retenidas, 2 para el porcentaje de $\mathrm{C}$ de $\mathrm{T}$ tomado en préstamos de bancos, 3 para el porcentaje de $C$ de $T$ tomado en préstamos de instituciones financieras no bancarias, 4 para el porcentaje de $\mathrm{C}$ de $\mathrm{T}$ comprado con crédito de proveedores 0 avances clientes ${ }^{5}$ y 5 para el porcentaje de $C$ de $T$ financiado por otros (prestamistas, amigos, parientes, etc.).

En el caso de la financiación de nuevos activos fijos se hicieron pruebas para las razones mencionadas anteriormente y para una razón financiera adicional que es la razón que indica el porcentaje de los nuevos activos fijos (AF) fondeados por contribución de los dueños o emisión de nuevo patrimonio, en el caso de que la empresa haya hecho adquisición de AF en el año fiscal anterior al de la fecha de la encuesta.

Posteriormente, para explorar la asociación entre el tipo y el grado de innovación empresarial y la financiación, teniendo en cuenta la influencia de otras variables que pueden afectar este grado de asociación ${ }^{6}$ se utilizó un modelo de regresión logística (Anderson et al., 2009, p. 679) cuya ecuación es la siguiente:

4 La prueba Mann-Whitney, también conocida como Mann-Whitney-Wilcoxon (MWW), es una prueba no paramétrica que no requiere que los datos sean medidos a un nivel de intervalo ni tampoco que las poblaciones tengan distribuciones normales. El único requerimiento es que la escala de medición sea ordinal. Por esta razón la prueba no es sobre la diferencia de las medias sino sobre si las poblaciones son idénticas (Anderson, Sweeney y Williams, 2009, pp. 825-830).

5 Nos referimos a esta fuente de financiación con el nombre de "crédito de proveedores".

6 Para seleccionar estas variables se tomaron en cuenta principalmente los resultados de los estudios de Ayyagari et al. (2011), Hall y Lerner (2010); García et al. (2013) y Barona y Rivera (2012 y 2013). La descripción de todas las variables independientes se presenta en la Tabla A1. En las regresiones logísticas empleadas se supuso que la decisión de la empresa sobre adelantar uno de los varios tipos de innovación es independiente de la decisión de hacer otro tipo de innovacion, lo cual no es estrictamente cierto pues las empresas pueden decidir hacer de manera simultánea todos los tipos de innovacion que deseen. Este supuesto demanda precaución al interpretar los resultados derivados de las regresiones logísticas que se describen en esta sección. 


$$
E(y)=\frac{e^{\beta_{0}+\beta_{1} X_{1}+\beta_{1} X_{1}+} \beta_{2} X_{2}+\cdots+\beta_{p} X_{p}}{1+e^{\beta_{0}+\beta_{1} X_{1}+\beta_{1} X_{1}+} \beta_{2} X_{2}+\cdots+\beta_{p} X_{p}}
$$

$y=$ variable independiente que indica el valor del índice agregado de innovación (IAI) o un tipo o nivel de innovación?.

$\mathrm{x}_{1}=$ porcentaje de financiación que representan los recursos internos/utilidades retenidas.

$\mathrm{X}_{1 \mathrm{~A}}=$ porcentaje de financiación que representan los recursos provenientes de contribución de los dueños o emisión de nuevo patrimonio.

$x_{2}=$ porcentaje de financiación que representan los préstamos bancarios.

$x_{3}=$ porcentaje de financiación que representan los préstamos de instituciones financieras no bancarias.

$\mathrm{x}_{4}=$ porcentaje de financiación que representa el crédito de proveedores / avances de clientes.

$\mathrm{x}_{5}=$ porcentaje de financiación que representa la fuente "otros".

$\mathrm{x}_{6}=$ respuesta a pregunta de si considera la financiación como obstáculo.

$x_{7}=$ logaritmo natural de edad en años.

$\mathrm{x}_{8}=$ respuesta a pregunta de si tiene o no cuenta bancaria.

$x_{9}=$ variable ficticia estatus legal de la empresa (responsabilidad de los dueños).

$\mathrm{x}_{10}=$ respuesta a pregunta de si se tiene inversión extranjera.

El primer paso en este análisis es someter a prueba la significancia de la regresión:

$H_{0}: \beta_{1}=\beta_{2}=\ldots . .=\beta_{9}=0$

$H_{a}$ : uno o más de los parámetros no son iguales a cero.

Siguiendo a Anderson et al. (2009, pp. 665-672), para someter a prueba esta hipótesis se empleó un test estadístico G: Si la hipótesis es cierta, la distribución muestral de G sigue una distribución chi-cuadrado con un número de grados de libertad igual al número de variables independientes en el modelo.

7 Siguiendo a Carayannis y Provance (2008) se construye un IAI con base en las respuestas dadas a las preguntas en el survey. El IAI puede asumir los valores 1 a 6 , en donde el 1 corresponde a las empresas más innovadoras y el 6 a las menos innovadoras (empresas que reportaron no hacer innovaciones, ni desembolsos en I+D, ni tener patentes reportadas). Ayyagari et al. (2011) utilizan un índice similar para analizar la innovación de las firmas en mercados emergentes. 
Si la prueba $G$ muestra que el modelo general es significativo, se puede usar una prueba $Z$ para determinar si cada una de las variables independientes del modelo hace una contribución significativa al mismo. Para las variables independientes Xi estas hipótesis son:

$H_{0}: \beta i=0$

$H_{a}: \beta i \neq 0$

Si la hipótesis nula anterior es cierta, el valor del coeficiente estimado dividido por su error estándar sigue una distribución de probabilidad estándar normal.

Para examinar la bondad de ajuste del modelo se emplearon los coeficientes Pearson y Hosmer-Lemeshow. En este caso el modelo se rechaza si los valores $p$ son bajos (en nuestro caso, por debajo de 0,05$)^{8}$.

Inicialmente se corrieron seis regresiones logísticas binarias para examinar las relaciones de cada uno de los tres tipos de innovación contempladas en el WBES con la importancia de las fuentes de financiación (variables $\mathrm{x}_{1} \mathrm{a} \mathrm{x}_{5}$ ) y las características de las empresas representadas en las variables $\mathrm{x}_{6}$ a $\mathrm{x}_{10}$ en el modelo descrito antes. En estas regresiones se tomaron como variables dependientes cada una de las respuestas dadas a la realización de alguna forma de innovación específica (preguntas DA, DB o DC de la Tabla 1). Estas variables en cada caso se codificaron con el valor cero (0) si la respuesta era que la empresa no hacía innovación y con el valor uno (1) en cualquier otro caso.

Las primeras tres regresiones se corrieron empleando la información de las respuestas dadas a las preguntas sobre financiación del capital de trabajo. Posteriormente se corrieron regresiones logísticas, pero teniendo en cuenta las respuestas dadas a las preguntas sobre financiación de activos fijos. De acuerdo con la teoría económica se espera que estas regresiones suministren evidencia de la importancia fundamental de los recursos internos en la financiación de la innovación y den respuesta a la pregunta de si -como sugieren algunos estudios recientes- la deuda bancaria contribuye también de manera significativa a la financiación de esta actividad (Williamson, 1988; Hall y Lerner, 2010; Sierra et al., 2009; Kerr y Nanda, 2014).

8 Para una discusión acerca del Hosmer-Lemeshow y sus limitaciones, ver University of Strathclyde (s.f.). 
A continuación, se corrieron dos regresiones logísticas adicionales en las que las variables dependientes fueron la respuesta a las preguntas DD ( $¿$ hizo el establecimiento desembolsos en actividades de I+D) y DL (¿tiene el establecimiento patentes registradas?).

Posteriormente se hizo un análisis complementario al anterior en el que, en vez de utilizar las respuestas dadas a la pregunta sobre realización de cada tipo de innovación, se utilizó un IAI. En este caso el modelo de análisis estadístico empleado fue una regresión logística ordenada9 .

Finalmente, para examinar la relación entre el monto invertido en $I+D$ y las variables $X_{1}$ a $X_{10}$ se corrió una regresión lineal múltiple. En todas las regresiones corridas, si alguno de los coeficientes $\beta_{1}$ a $\beta_{10}$ resulta significativamente diferente de cero, la hipótesis de que la variable independiente específica no está relacionada con el respectivo tipo de innovación se rechaza, y se considera evidencia a favor de la existencia de relación cuando se toma en consideración, simultáneamente, la presencia de todas las variables indicadas en las regresiones.

\section{Resultados}

La Tabla 3 contiene información sobre los promedios de las respuestas dadas en relación con las diferentes fuentes de financiación empleadas en los casos de inversión en capital de trabajo y en nuevos activos fijos ${ }^{10}$, tanto para el total de empresas de la muestra, como para las empresas clasificadas como innovadoras y no innovadoras.

Se destacan los siguientes aspectos de los patrones de financiamiento: primero, las utilidades retenidas constituyeron la fuente de financiación individual más importante de las empresas de la muestra, su porcentaje promedio osciló entre un mínimo de 36,6\% y un máximo de $44,9 \%{ }^{11}$. La prueba estadística realizada indica que la hipótesis de que los dos tipos de empresas (innovadoras y no innovadoras) usaron esta fuente de financiación de manera igual no se puede aceptar (considerando un nivel de significancia del 10\%). Segundo, se advierte que las empresas innovadoras y las no innovadoras también usan

9 Una discusión de las regresiones Ordered Logit and Ordered Probit se encuentra en Wooldridge (2002).

10 Como en el WBES solo se hicieron preguntas relacionadas con la financiación de activos fijos a las empresas que reportaron haber hecho este tipo de inversiones, el número de respuestas se redujo a 259.

11 Solamente en el caso de la financiación del capital de trabajo, para el grupo de empresas no innovadoras, esta fuente cedió su preeminencia a otra fuente (crédito/avance de proveedores, que obtuvo 37,3\%). 


\section{Tabla 3}

Fuentes de financiación del total de empresas. Valores promedio

\begin{tabular}{|c|c|c|c|c|c|}
\hline & $\begin{array}{c}\text { Total } \\
\text { empresas }\end{array}$ & $\begin{array}{c}\text { No } \\
\text { innovadoras }\end{array}$ & Innovadoras & $\begin{array}{c}\text { Valor } \mathrm{p} \text { en } \\
\text { prueba } \mathrm{MW}+\end{array}$ & Significancia \\
\hline $\begin{array}{l}\text { Financiación de } C \text { de } \mathrm{T} \mathrm{N}=869 \\
\% \text { de PP de } C \text { de T financiado por: }\end{array}$ & $\%$ & $\%$ & $\%$ & & \\
\hline Fuentes internas/utilidades retenidas & 37,5 & 36,6 & 40,3 & 0,0624 & * \\
\hline Bancos & 21,2 & 20,0 & 23,5 & 0,0347 & ** \\
\hline Instituciones financieras no bancarias & 1,5 & 1,8 & 0,8 & 0,4119 & \\
\hline Crédito /avances de proveedor / cliente & 35,3 & 37,3 & 30,9 & 0,0080 & $* * *$ \\
\hline Otros & 4,3 & 4,2 & 4,6 & 0,6420 & \\
\hline $\begin{array}{l}\text { Financiación de nuevos AF N= } 259 \\
\% \text { PP de nuevos AF financiado por: }\end{array}$ & $\%$ & $\%$ & $\%$ & & \\
\hline Fuentes internas / utilidades retenidas & 43,6 & 44,9 & 41,6 & 0,3623 & \\
\hline $\begin{array}{l}\text { Contribución de los dueños o emisión de } \\
\text { nuevas acciones }\end{array}$ & 4,2 & 3,4 & 5,3 & 0,3031 & \\
\hline Bancos & 32,8 & 31,3 & 35,1 & 0,1699 & \\
\hline Instituciones financieras no bancarias & 4,4 & 4,8 & 3,8 & 0,6891 & \\
\hline Crédito de proveedor o avances de cliente & 11,9 & 13,6 & 9,4 & 1,0000 & \\
\hline Otros & 3,1 & 1,9 & 4,9 & 0,5097 & \\
\hline
\end{tabular}

MW es la prueba Mann-Whitney, también conocida como Mann-Whitney-Wilcoxon (MWW) descrita antes en el texto.

Significativa: * al $10 \%,{ }^{* *}$ al $5 \% \mathrm{y} * * *$ al $1 \%$.

Fuente: elaboración propia, con base en Enterprise Survey-Raw Data (2010).

de manera diferente la financiación bancaria y el crédito de proveedores; los resultados sugieren que esta última fuente de financiación parece tener mayor importancia en las empresas no innovadoras que en las innovadoras ${ }^{12}$.

El hallazgo de que la principal fuente de financiación del total de empresas es la interna y de que las innovadoras parecen emplear más intensivamente esta fuente de financiación es consecuente con teorías modernas de financiación que consideran que debido a problemas como el riesgo moraly la selección adversa, la fuente de financiación más idónea para financiar la innovación son las utilidades retenidas (Hally Lerner, 2010; Williamson,

12 En la prueba MW el uso diferente de la fuente de financiación crédito de proveedores para financiar capital de trabajo fue la que alcanzó un nivel de significancia más bajo (al 1\%). Las empresas no innovadoras parecen usar esta fuente de manera más intensiva que las innovadoras. 
1996). Las cifras de la Tabla 3 también sugieren que la importancia de la financiación interna es mayor en el caso de que la inversión sea en nuevos activos fijos que en el caso de inversión en capital de trabajo, lo cual coincide con lo que sugiere la teoría financiera de buscar congruencia entre la duración de los activos y la de las fuentes de financiación. Cuando se examinan las diferencias entre las fuentes de financiación del capital de trabajo y las de los nuevos activos fijos se observa que en el caso de la financiación del capital de trabajo la segunda fuente de financiación es el crédito de proveedores y la tercera los bancos; este orden se altera en el caso de la financiación de nuevos activos fijos en el que la fuente proveedores pierde importancia de manera considerable, lo cual es coherente con la teoría financiera, ya que los créditos con proveedores tienden a ser de duración bastante menor $(30,60,90$ o 180 días) que los créditos bancarios, que con frecuencia se obtienen por períodos de hasta cinco años.

En la Tabla 4 se presentan los resultados de las regresiones logísticas que se corrieron para identificar relaciones entre la realización de tipos de innovaciones y diez variables que teóricamente podrían estar relacionadas con estas. Los datos de la Tabla indican que las cuatro regresiones resultaron significativas al $1 \%$ y arrojaron indicadores de bondad de ajuste razonables, por lo cual se concluyó que la hipótesis de que el modelo no es válido, no se puede aceptar.

Con respecto a las variables predictoras de la realización de innovaciones, los datos mostraron que en las cuatro regresiones resultó altamente significativa (en tres ocasiones al 1\%) la relación positiva entre la realización de innovación y la respuesta de que la financiación se percibe como un obstáculo. En lo que tiene que ver con la financiación del capital de trabajo la variable edad mostró relación positiva significativa con las respuestas afirmativas a las preguntas DA y DB (al 5\%) pero no con respecto a la DC. En la regresión que hace referencia a la financiación de los activos fijos, esta variable resultó significativa, pero solo al $10 \%$. Con respecto a las variables que reflejan la importancia relativa de fuentes de financiación se nota que tratándose de la financiación del capital de trabajo, en el caso de la respuesta DA, ninguna variable resultó significativa al 1\% y solo una al $5 \%$ (la financiación por crédito de proveedores). En el caso de la respuesta a $D B$ resultaron significativos al $5 \%$ la variable que mide la importancia relativa de la fuente de financiación préstamos de instituciones financieras no bancarias, la edad en años y el percibir la financiación como un obstáculo. En el caso de la financiación de activos fijos (que tiene un tamaño de muestra bastante menor), la variable que indica la percepción como obstáculo, la que indica si la empresa tiene inversión extranjera y la edad resultaron significativas al $1 \%, 5 \%$ y $10 \%$, respectivamente. 


\section{Tabla 4}

Relación entre tipo de innovación realizada, fuentes de financiación y características de las empresas. Resultados de regresión logística binaria

\begin{tabular}{|c|c|c|c|c|}
\hline \multirow{4}{*}{ Variables predictoras } & \multicolumn{3}{|c|}{$\begin{array}{l}\text { Financiación del capital de trabajo } \\
\qquad \mathrm{N}=869\end{array}$} & \multirow{2}{*}{$\begin{array}{c}\begin{array}{c}\text { Financiación } \\
\text { activos fijos } \\
\text { N }=259\end{array} \\
\text { DA }\end{array}$} \\
\hline & DA & DB & DC & \\
\hline & $\begin{array}{c}\mathrm{Si}=203 \\
\mathrm{No}=666\end{array}$ & $\begin{array}{c}\mathrm{Si}=366 \\
\mathrm{No}=503\end{array}$ & $\begin{array}{c}\mathrm{Sí}=188 \\
\mathrm{No}=681\end{array}$ & $\begin{array}{c}\mathrm{Si}=115 \\
\mathrm{No}=144\end{array}$ \\
\hline & Significancia & Significancia & Significancia & Significancia \\
\hline Constante & 0,218 & 0,558 & 0,765 & 0,999 \\
\hline $\begin{array}{l}\text { X1 Porcentaje de financiación que representan } \\
\text { los recursos internos / utilidades retenidas }\end{array}$ & 0,093 * & 0,975 & 0,723 & 0,622 \\
\hline $\begin{array}{l}\text { X2 Porcentaje de financiación que representan } \\
\text { los préstamos bancarios }\end{array}$ & 0,062 * & 0,677 & 0,686 & 0,881 \\
\hline $\begin{array}{l}\text { X3 Porcentaje de financiación que representan } \\
\text { los préstamos de instituciones financieras no } \\
\text { bancarias }\end{array}$ & 0,051 * & $0,045 * *$ & 0,384 & 0,440 \\
\hline $\begin{array}{l}\text { X4 Porcentaje de financiación que representa } \\
\text { el crédito de proveedores /avance de clientes }\end{array}$ & $0,022 * *$ & 0,380 & 0,251 & 0,903 \\
\hline $\begin{array}{l}\text { X1A Porcentaje de financiación por contribución } \\
\text { de los dueños / emisión de nuevas acciones }\end{array}$ & & & & 0,833 \\
\hline X6 Financiación como obstáculo & $0,000 * * *$ & $0,010 * *$ & $0,001 * * *$ & $0,000 * * *$ \\
\hline X7 Logaritmo natural de edad en años & $0,003 * *$ & 0,033 ** & 0,629 & 0,059 * \\
\hline $\begin{array}{l}\text { X8 Variable ficticia sobre si tiene o no cuenta } \\
\text { bancaria }\end{array}$ & 0,866 & 0,686 & 0,242 & 0,999 \\
\hline $\begin{array}{l}\text { X9 Variable ficticia estatus legal de la empresa } \\
\text { (responsabilidad de los dueños) }\end{array}$ & 0,155 & 0,976 & 0,827 & 0,188 \\
\hline X10 Variable ficticia para inversión extranjera & $0,002 * *$ & 0,437 & 0,236 & $0,004 * *$ \\
\hline Log-verosimilitud & $-452,048$ & $-580,473$ & $-442,889$ & $-165,282$ \\
\hline \multicolumn{5}{|l|}{ Probar que todas las pendientes son cero: } \\
\hline$G=$ & 40,666 & 22,055 & 21,871 & 25,232 \\
\hline $\mathrm{GL}=$ & 9 & 9 & 9 & 10 \\
\hline Valor $p=$ & 0,000 & 0,009 & 0,009 & 0,005 \\
\hline Pruebas de bondad de ajuste & 0,349 & 0,44 & 0,382 & 0,294 \\
\hline Pearson Desviación & 0,081 & 0 & 0,222 & 0,000 \\
\hline Hosmer-Lemeshow & 0,328 & 0,218 & 0,426 & 0,918 \\
\hline
\end{tabular}

Variable DA: ¿Productos nuevos/significativamente mejorados al mercado del establecimiento?

Variable DB: ¿Procesos nuevos/significativamente mejorados para producir/Suministros productos?

Variable DC: ¿Procesos nuevos / significativamente mejorados también nuevos para su industria?

* Significancia al 10\%, ** Significancia al 5\%, ** Significancia al 1\%.

Fuente: elaboración propia con base en Enterprise Survey-Raw Data (2010). 
En relación con la respuesta de haber hecho desembolsos para I+D y la tenencia de patentes, en la Tabla 5 se muestra que las regresiones son significativas y que las variables asociadas con esta son: la edad y la inversión extranjera (esta última solo significativa al $10 \%$ en el caso de la pregunta sobre haber hecho desembolsos para actividades de I+D).

\section{Tabla 5}

Relación entre inversión en actividades de I+D y tener patentes con las fuentes de financiación y características de las empresa. Regresión logística binaria

\begin{tabular}{|c|c|c|}
\hline \multirow{3}{*}{ Variables predictoras } & Variable DD & Variable DL \\
\hline & $\begin{array}{c}S_{1}^{1}=362 \\
N_{0}=507\end{array}$ & $\begin{aligned} \mathrm{Si} & =187 \\
\mathrm{No} & =682\end{aligned}$ \\
\hline & Significancia & Significancia \\
\hline Constante & 0,080 * & 0,144 \\
\hline $\begin{array}{l}\text { X1 Porcentaje de financiación que representan los recursos internos / utilidades } \\
\text { retenidas }\end{array}$ & 0,703 & 0,232 \\
\hline X2 Porcentaje de financiación que representan los préstamos bancarios & 0,960 & 0,329 \\
\hline $\begin{array}{l}\text { X3 Porcentaje de financiación que representan los préstamos de instituciones fi- } \\
\text { nancieras no bancarias }\end{array}$ & 0,783 & 0,130 \\
\hline $\begin{array}{l}\text { X4 Porcentaje de financiación que representa el crédito de proveedores /avance } \\
\text { de clientes }\end{array}$ & 0,116 & 0,288 \\
\hline \multicolumn{3}{|l|}{$\begin{array}{l}\text { X1A Porcentaje de financiación por contribución de los dueños / emisión de nuevas } \\
\text { acciones }\end{array}$} \\
\hline X6 Financiación como obstáculo & 0,243 & 0,114 \\
\hline X7 Logaritmo natural de edad en años & $0,000 * * *$ & $0,000 * * *$ \\
\hline X8 Variable ficticia sobre si tiene o no cuenta bancaria & 0,468 & 0,857 \\
\hline X9 Variable ficticia estatus legal de la empresa (responsabilidad de los dueños) & 0,870 & 0,663 \\
\hline X10 Variable ficticia para inversión extranjera & 0,059 * & $0,028 * *$ \\
\hline Log-verosimilitud & $-570,780$ & $-431,811$ \\
\hline \multicolumn{3}{|l|}{ Probar que todas las pendientes son cero: } \\
\hline $\mathrm{G}=$ & 38,821 & 41,445 \\
\hline $\mathrm{GL}=$ & 9,000 & 9,000 \\
\hline Valor $p=$ & 0,000 & 0,000 \\
\hline Pruebas de bondad de ajuste & 0,314 & 0,321 \\
\hline Pearson Desviación & 0,000 & 0,332 \\
\hline Hosmer-Lemeshow & 0,839 & 0,174 \\
\hline
\end{tabular}

Variable DD: ¿Hizo el establecimiento desembolsos en actividades de I+D en su interior?

Variable DL: ¿Tiene el establecimiento patentes registradas en su país?

* Significancia al $10 \%$; * Significancia al $5 \%$; ** * Significancia al $1 \%$

Fuente: elaboración propia con base en Enterprise Survey-Raw Data (2010). 
En la Tabla 6 se resumen los resultados de la regresión logística ordenada en la que la variable dependiente es un índice agregado de innovación que podía asumir los valores 1 a 6 (1 para las empresas más innovadoras y 6 para las menos innovadoras). Se observa que la regresión es significativa $(p=0,000)$ y que las variables predictoras con signo positivo significativas al $1 \%$ resultaron ser financiación como obstáculo $(X 6)$, edad $(X 7)$ e inversión extranjera (X10). Igualmente resultó significativo a un menor nivel (al 10\%), pero denotando relación inversa, el porcentaje de financiación proveniente de crédito de proveedores $(\mathrm{X} 4)$.

\section{Tabla 6}

Resultados de la regresión logística ordenada. Relación índice agregado de innovación y 10 variables independientes

Regresión logística ordinal: Y6 indcecom vs. X1-EX; X2-EY; ...

Función de enlace: Normit

Información de respuesta

\begin{tabular}{rcc}
\hline Variable & Valor & Conteo \\
\hline Y6 indcecom & 1 & 39 \\
2 & 91 \\
3 & 108 \\
4 & 145 \\
5 & 133 \\
6 & 353 \\
TOTAL & 869 \\
\hline
\end{tabular}

Tabla de regresión logística

\begin{tabular}{lcccc}
\hline \multicolumn{1}{c}{ Predictor } & Coef & SE Coef & Z & P \\
\hline Const (1) & $-2,17935$ & 0,61041 & $-3,57$ & 0,000 \\
Const(2) & $-1,50141$ & 0,60765 & $-2,47$ & 0,013 \\
Const(3) & $-1,05231$ & 0,60688 & $-1,73$ & 0,083 \\
Const(4) & $-0,58641$ & 0,60645 & $-0,97$ & 0,334 \\
Const(5) & $-0,18747$ & 0,60629 & $-0,31$ & 0,757 \\
X1-EX & $-0,00277$ & 0,00289 & $-0,96$ & 0,339 \\
X2-EY & $-0,00145$ & 0,00302 & $-0,48$ & 0,632 \\
X3-EZ & $-0,009836$ & 0,00638 & $-1,54$ & 0,123 \\
X4-FA & $-0,005695$ & 0,00295 & $-1,93$ & 0,054 \\
X6 fincomoobs & 0,082878 & 0,03060 & 2,71 & 0,007 \\
X7 loged & 0,225285 & 0,05214 & 4,32 & 0,000
\end{tabular}




\begin{tabular}{lcccc}
\hline \multicolumn{1}{c}{ Predictor } & Coef & SE Coef & Z & P \\
\hline x8cntab & $-0,009149$ & 0,54779 & $-0,02$ & 0,987 \\
X9sttusl & $-0,055613$ & 0,11423 & $-0,49$ & 0,626 \\
X10 INVEX & 0,318895 & 0,12267 & 2,60 & 0,009 \\
\hline
\end{tabular}

Log-verosimilitud $=-1.357,393$

Probar que todas las pendientes son cero: $G=42,971, G L=9$, valor $P=0,000$

Pruebas de bondad del ajuste

\begin{tabular}{lccc}
\hline \multicolumn{1}{c}{ Método } & Chi-cuadrada & $\mathrm{GL}$ & $\mathrm{P}$ \\
\hline Pearson & $4.223,98$ & 4.221 & 0,484 \\
Desviación & $2.674,92$ & 4.221 & 1,000 \\
\hline
\end{tabular}

Medidas de asociación

(Entre la variable de respuesta y las probabilidades pronosticadas)

\begin{tabular}{lcclc}
\hline \multicolumn{1}{c}{ Pares } & Número & Porcentaje & \multicolumn{2}{c}{ Medidas de resumen } \\
\hline Concordante & 167.525 & 58,7 & D de Somers & 0,18 \\
Discordante & 116.124 & 40,7 & Gamma de Goodman-Kruskal & 0,18 \\
Empates & 1.537 & 0,5 & Tau-a de Kendall & 0,14 \\
Total & 285.186 & 100,0 & & \\
\hline
\end{tabular}

Fuente: elaboración propia, utilizando el programa MINITAB con base en Enterprise Survey-Raw Data (2010).

En la Tabla 7 se muestran los resultados de una regresión lineal múltiple, utilizando el método paso a paso, con en el monto invertido en I+D como variable dependiente y como independientes las diez variables incluidas en las otras regresiones. Los resultados confirman lo encontrado en el caso de las regresiones binarias con respecto a la correlación significativa de la investigación y desarrollo con la edad y la inversión extranjera; empero, se observa que una variable no detectada como significativa en la regresión binaria: la importancia relativa de la fuente de financiación préstamo de instituciones no bancarias, se torna significativa (al 5\%).

\section{Discusión}

Los resultados obtenidos indican que el tipo de innovación más frecuente fue procesos nuevos/significativamente mejorados para producir/suministrar productos; le siguen en orden de frecuencia los productos nuevos/significativamente mejorados al mercado del establecimiento y, finalmente, los procesos nuevos/significativamente mejorados, también, nuevos para su industria. 


\section{Tabla 7}

Relación monto de I+D en pesos y las diez variables independientes. Resultados de la regresión lineal múltiple paso a paso

\begin{tabular}{|c|c|c|c|}
\hline \multicolumn{4}{|c|}{ La respuesta es $\mathrm{Y} 7$ en diez predictores, con $\mathrm{N}=869$} \\
\hline Paso & 1 & 2 & 3 \\
\hline Constante & 79.691 .933 & -375.728 .677 & -405.477 .897 \\
\hline X10 Inv. Extranjera & 901.346 .614 & 850.648 .294 & 856.405 .118 \\
\hline Valor T & 5,71 & 5,36 & 5,41 \\
\hline Valor P & 0,00 & 0,00 & 0,00 \\
\hline X7 log edad & & 158.769 .937 & 160.462 .121 \\
\hline Valor T & & 2,43 & 2,47 \\
\hline Valor $\mathrm{P}$ & & 0,02 & 0,01 \\
\hline $\mathrm{X} 3 \mathrm{EZ}$ & & & 16.366 .359 \\
\hline Valor T & & & 2,37 \\
\hline Valor $\mathrm{P}$ & & & 0,02 \\
\hline$S$ & 1.404.196.380 & 1.400.223.117 & 1.396.516.050 \\
\hline R-cuad. & 3,62 & 4,28 & 4,89 \\
\hline R-cuad.(ajustado) & 3,51 & 4,06 & 4,56 \\
\hline
\end{tabular}

Fuente: elaboración propia, utilizando el programa MINITAB con base en Enterprise Survey-Raw Data (2010).

Al considerar la muestra total de empresas, innovadoras y no innovadoras, en el Survey se halló en primer lugar que la fuente de financiación más importante, tanto en el caso de inversión en nuevos activos fijos, como en inversión en capital de trabajo, fue el recurso interno (utilidad retenida); el porcentaje promedio de las respuestas dadas por los encuestados osciló entre un mínimo de 36,6\% y un máximo de 44,9\%. Las pruebas estadísticas sobre el uso de esta fuente de financiación por parte de las empresas innovadoras y no innovadoras mostraron que la hipótesis de que se hace un uso diferente de esta fuente de financiación por parte de ambos tipos de poblaciones no se puede rechazar. Este hallazgo está en armonía con la conclusión a que llegan Hall y Lerner (2010), quienes afirman que en presencia de asimetría de información, el conflicto de interés entre el agente y el principal hace que la financiación por nueva deuda o nuevo patrimonio sea relativamente más costosa para la $I+D$, que para la inversión ordinaria: “Juntos estos argumentos sugieren un rol importante para las utilidades retenidas en la decisión de I+D, independientemente de su valor como señal de rentabilidad futura" (p. 618). Hogan 
y Hutson (2005) y Ughetto (2008) llegan a conclusiones similares sobre la importancia de la retención de las ganancias para financiar las actividades de innovación.

Los análisis estadísticos muestran que las empresas innovadoras parecen hacer un uso más intensivo de la fuente préstamos bancarios que las no innovadoras; igualmente sugieren que la fuente crédito de proveedores es también utilizada de manera diferente por las empresas innovadoras y las no innovadoras, en tanto el promedio mostrado en la Tabla 3 indica que las no innovadoras utilizan más intensivamente esta fuente de financiación.

Cuando en el análisis de la relación entre la innovación y las fuentes de financiación se tiene en cuenta la incidencia de otras variables como edad, naturaleza jurídica y respuestas a las preguntas de si las empresas ven la financiación como un obstáculo, tienen cuenta bancaria y tienen inversión extranjera, los modelos estadísticos empleados sugieren que las variables que muestran una asociación más fuerte con la realización de innovaciones, con el monto de recursos invertidos en I+D y con la tenencia de patentes, son la edad y la presencia de inversión extranjera. Adicionalmente los análisis multivariados muestran que las empresas que hacen algún tipo de innovación o invierten en I+D consideran en mayor grado que la financiación es un obstáculo, frente a las no innovadoras.

Comparación de los resultados con los de estudios similares en el país: Langebaek y Vásquez (2007) encuentran que hay una relación fuerte entre la innovación y las variables tamaño de la empresa, presencia de capital extranjero y el capital humano de la firma. Los estudios de Sierra et al. (2009) y Arbeláez y Parra (2011) también concluyen que en Colombia las empresas de mayor tamaño son más innovadoras que las de menor tamaño. Este resultado tiene particular interés para la política pública, específicamente en la definición del tipo de empresas hacia donde deberían dirigirse prioritariamente los recursos del Estado y en el establecimiento de estímulos tributarios y de otra índole para estimular la innovación. Estudios económicos pioneros sobre este tema sugieren que las firmas pequeñas tienden a ser más innovadoras que las grandes ya que en estas últimas los costos de agencia tienden a ser mayores y más difíciles de controlar (Holmstrom, 1989). No obstante, investigaciones como la de Lerner (2012, cap. 3) muestran que algunas grandes corporaciones han encontrado formas relativamente eficientes de controlar estos costos mediante la descentralización y la innovación abierta. Sierra et al. (2009) encuentran una alta correlación entre el tamaño de las empresas y el grado de innovación, siendo las grandes las más innovadoras (p. 192); adicionalmente indican que las principales razones que aducen las empresas más pequeñas para no innovar es que consideran que "la innovación no es rentable o innecesaria; también, aunque con 
menos fuerza aducen que no lo hacen por falta de recursos financieros" (pp. 188-189). En este punto es pertinente hacer referencia al trabajo de Otálora, Hurtado y Quimbay (2009) en el que, utilizando el enfoque de redes complejas, indagan sobre el interés que los empresarios del sector industrial manufacturero colombiano tienen para acceder a la financiación de actividades de investigación, desarrollo experimental e innovación $(\mathrm{I}+\mathrm{D}+\mathrm{i})$ y sobre la visibilidad de las fuentes de financiación; entre los hallazgos de este estudio se destaca que "los empresarios en promedio tienen poco interés o aversión por conocer fuentes de financiación" (p. 228). En la investigación de Sierra et al. (2009) los empresarios perciben el acceso a la financiación como algo que restringe su actividad innovadora y que el problema de financiación parece tener que ver tanto con el acceso a esta, como con su costo relativamente alto. Además afirman que sus análisis parecen confirmar la existencia del orden de jerarquías en la financiación de las empresas que adelantan proyectos de investigación. La base de datos utilizada en nuestro estudio no permitió hacer análisis acerca de la jerarquía de preferencias de fuentes de financiación por parte de empresarios.

El resultado de la prevalencia de los recursos propios y de los provistos por la banca privada encontrado en esta investigación coincide también con los resultados de Sierra et al. (2009) y de Barona, Rivera, Aguilera y Garizado (2014).

\section{Conclusiones}

La fuente de financiación más importante en el total de empresas de la muestra, tanto en el caso de inversión en nuevos activos fijos, como en inversión en capital de trabajo, fue el recurso interno (utilidad retenida). Las empresas innovadoras parecen usar un patrón de financiamiento diferente al de las no innovadoras, el cual se caracteriza por hacer (las innovadoras) un mayor uso de la fuente utilidades retenidas y de la financiación bancaria, y un menor uso del crédito de proveedores. La utilización de los recursos internos como principal fuente de recursos es consistente con la teoría económica que sostiene que la característica de este tipo de recursos hace que sean los más idóneos para financiar inversiones en innovación e I+D.

De otra parte, el hallazgo de la importancia del uso de la fuente de financiación bancaria es consistente con lo encontrado por otros estudios en Colombia, en países emergentes, y más recientemente, en Estados Unidos, país en el que el mercado accionario y del capital privado se encuentra más desarrollado; al respecto Kerr y Nanda (2014) anotan haber observado que el uso de la deuda en la financiación de la innovación fue “más prevalente 
de lo anticipado", asunto que guarda relación con ciertas limitaciones del mercado accionario en la financiación de la innovación, las cuales tienen que ver con presiones de corto plazo y limitaciones en el monitoreo (p. 4). En nuestro estudio se encontró que una fuente de recursos relativamente importante es el crédito de proveedores, algo relativamente poco estudiado en la literatura de la financiación de la innovación, pero que los empresarios deberían tener en cuenta en la planeación de los recursos necesarios para financiar sus proyectos de innovación (Robb y Robinson, 2014).

Los resultados de otros estudios en Colombia muestran una relación positiva entre la edad de la empresa y la realización de innovación; nuestro estudio llegó a una conclusión similar cuando el tamaño se considera como proxy de la edad. Este hallazgo merece ser explorado en mayor profundidad con nuevos datos, ya que existen sólidos argumentos de naturaleza organizacional para pensar que son las empresas jóvenes y de menor tamaño las que presentan las condiciones más propicias para la innovación (Holmstrom, 1989).

Combinando los hallazgos de que las empresas innovadoras usan más intensivamente fuentes internas/utilidades retenidas y créditos bancarios que las no innovadoras, con el hecho de que el porcentaje de los dueños/gerentes de empresas innovadoras que respondió que la financiación es un obstáculo, fue significativamente mayor que el que dio la misma respuesta en empresas no innovadoras, se puede conjeturar que, si bien las empresas innovadoras han recurrido a las fuentes internas y al crédito bancario para la realización de sus operaciones, dichas empresas consideran que tales fuentes han sido insuficientes para permitirles alcanzar el desarrollo potencial que sus dueños/gerentes consideran factible. El hallazgo descrito y mencionado antes, de una relación significativa entre el monto invertido en I+D y el uso de préstamos de instituciones no bancarias, sugiere que esta fuente pudo haber sido utilizada para subsanar tal insuficiencia.

La frecuencia con que las empresas innovadoras responden que la financiación ha sido un obstáculo, sugiere que el Estado colombiano debe tomar una postura más proactiva frente a este problema y mejorar las condiciones para que los empresarios cuenten con una variedad amplia de oportunidades para financiar actividades de innovación e investigación y desarrollo. Algunas acciones posibles, tratadas en detalle en Barona (2014), incluyen: i) mejorar la reglamentación de los fondos regionales y nacional de garantías para que el sistema bancario pueda ampliar su oferta de financiación sin un deterioro grande en su exposición al riesgo; ii) mejorar la reglamentación que permita una actividad más efectiva de fondos nacionales e internacionales de capital de riesgo y capital privado en el país; iii) incentivar tributariamente la realización de este tipo de 
actividades; iv) promover el desarrollo de la financiación comunitaria (crowdfunding, 0 mecanismos similares basados en las TIC), la cual ha mostrado resultados muy exitosos en los últimos años en otras partes del mundo (Dittmer, McCahery y Vermeulen, 2013).

En cuanto a las limitaciones del presente estudio se deben mencionar, en primer lugar, las características de la muestra empleada, la cual estuvo conformada por un número relativamente pequeño de empresas, en las que las de menor tamaño estuvieron sub-representadas, lo mismo que las pertenecientes a sectores económicos diferentes al manufacturero. Además, se nota que los análisis multivariados presentados, aunque con significancia estadística, arrojaron en algunos casos bajos coeficientes de determinación $\left(R^{2}\right)$, lo cual indica que porcentajes importantes de la variación en las variables dependientes de las regresiones (realización de innovación, desembolsos en I+D y tenencia de patentes) son explicados por variables no incluidas en las regresiones. Estas limitaciones indican que los resultados aquí presentados deben tenerse como preliminares.

Futuras investigaciones podrían eliminar en el análisis el supuesto de que las decisiones sobre innovación en el ámbito empresarial son independientes entre sí; esto implica emplear técnicas econométricas diferentes a las regresiones logísticas utilizadas en este artículo. Futuras investigaciones podrían también emplear bases de datos que corrijan algunas de las deficiencias ya anotadas. El uso de la información contenida en las Encuestas EDIT, que viene adelantando periódicamente el Departamento Administrativo Nacional de Estadística (DANE), es una alternativa interesante y que debería emplearse más intensivamente para investigar también otros aspectos relacionados con la innovación y la investigación y desarrollo. Además de estudios econométricos que posibiliten análisis más robustos y generalizaciones más amplias, los autores consideran también de gran trascendencia realizar estudios usando métodos cualitativos de recolección y análisis de información, como las encuestas y los estudios de casos (Yin, 2003), los cuales podrían explorar en mayor profundidad el hallazgo reportado en este estudio de que las empresas innovadoras usan mezclas de fuentes de financiación diferentes de las no innovadoras y también deberían develar y analizar la forma en la que empresarios e intermediarios financieros actuales o potenciales en Colombia perciben el problema y conciben su superación. Finalmente, una comprensión más profunda del problema de la financiación de la innovación en el país hace necesario el estudio de los aspectos institucionales relacionados con las características de la oferta de recursos financieros a la empresa. Algunos trabajos previos han abordado este estudio (ej.: Sierra et al., 2009); no obstante parece urgente adelantar nuevas investigaciones sobre el tema; para esto el 
enfoque adoptado por estudios recientes de los Sistemas Nacionales de Innovación lucen particularmente atractivos (Soete, Verspagen y Weel, 2010; Kahn, Melo y Matos, 2014).

\section{Referencias}

Anderson, D., Sweeney, D., and Williams, T. (2009). Statistics for Business and Economics (10 ed.). Mason, OH, USA: Thomson South-Western.

Arbeláez, M., and Parra, M. (2011). Innovation, R\&D investment and productivity in Colombian Firms. IDB. Working Paper Series No. IDB-WP-251.

Arrow, K. (1962). Economic welfare and the allocation of recourses for invention. The rate and direction on inventive activity. Princeton, NJ: Princeton University Press.

Ayyagari, M., Demirgünc-Kunt, A., and Maksimovic, V. (2011). Firm innovation in emerging markets: The role of finance, governance and competition. Journal of Financial and Quantitative Analysis, 46, 1545-1580.

Barona, B. (2014). Sistemas financieros incluyentes (SFI). Las microfinanzas. Una herramienta de politica pública para la disminución de la pobreza. Boletín POLIS 14, 13-16.

Barona, B. y Rivera, J. (2012). Finanzas emprendedoras en Colombia. Conceptos y resultados de algunos estudios. Cali: Universidad del Valle.

Barona, B.y Rivera, J. (2013). Financiación de nuevas empresas: comparación de las fuentes de financiación en Colombia y Chile. Cuadernos de Administración, 26 (46), 11-36.

Barona, B., Rivera, J., Aguilera, I. y Garizado, P. (2014). La financiación de la innovación de las empresas del sector manufacturero en Colombia. Un análisis de los resultados de la EDIT IV. Documento no publicado. Cali: Universidad del Valle.

Carayannis, E., and Provance, M. (2008). Measuring firm innovativeness: Towards a composite innovation index built on firm innovative posture, propensity and performance attributes. International Journal of Innovation and Regional Development, 1, 90-107.

Carpenter, R., and Petersen, B. (2002). Capital market imperfections, high-tech investment, and new equity financing. The Economic Journal, 112, F54-F72.

Dittmer, J., McCahery, J., and Vermeulen, E. (2013). The 'new' venture capital cycle and the role of governments. The emergence of collaborative funding models and platforms. Tilburg Law School Research Paper No. 021/2013 Lex Research Topics in Corporate Law y Economics. Working Paper 2013-7. Recuperado de: http://ssrn.com/abstract=2357771.

Enterprise Survey-Raw Data (2010). Recuperado de: http://www.enterpriseSurveys.org/Data. García, D., Barona, B. y Madrid, A. (2013). Financiación de la innovación en las mipymes iberoamericanas. Estudios Gerenciales, 29 (126), 12-16.

Hall, B. (2002). The financing of research and development. Oxford Review of Economic Policy, $18(1), 35-51$. 
Hall, B., and Lerner, J. (2010). The financing of R\&D and innovation. In: B. Hall and N. Rosenberg (eds.), Handbook in Economics (chap.14). Amsterdam: Elsevier.

Hogan, T., and Hutson, E. (2005). Capital structure in new technology -based firms: Evidence from the Iris software sector. Global Finance Journal, 15, 369-387.

Holmstrom, B. (1989). Agency costs and innovation. Journal of Economic Behavior and Organization, 12, 305-327.

Kahn, M., Melo, L., and Matos, M. (eds.) (2014). Financing innovation. London: Routledge.

Kerr, W., and Nanda, R. (2014). Financing innovation. Harvard Business Scholl Working Paper 15-034.

Langebaek, A. y Vásquez, D. (2007). Determinantes de la actividad innovadora en la industria manufacturera colombiana. Working Paper 433. Bogotá: Banco de la República.

Léger, A., and Swaminathan, S. (2007). Innovation theories: Relevance and implications for developing country innovation. Berlin: German Institute for Economic Research.

Lerner, J. (2012). The architecture of innovation: The economics of creative organizations. Boston: Harvard Business School Publishing Corporation.

Lozano, R. (2013, 5 de septiembre). Grandes firmas duran 18 años, según un estudio. Recuperado de: http://www.portafolio.co/negocios/duracion-las-Pymes-colombia

Nanda, R., and Rhodes-Kropf, M. (2013a). Financing Risk and Innovation. Working Paper 11-013. Harvard Business School Entrepreneurial Management.

Nanda, R., and Rhodes-Kropf, M. (2013b). Innovation and the Financial Guillotine. NBER Working Papers 19379, National Bureau of Economic Research, Inc.

O'Sullivan, M. (2005) Finance and innovation. In: J. Fagerberg, D. Mowery, and R. Nelson (eds.), The Oxford handbook of innovation. 0xford: 0xford University Press.

Otálora, D., Hurtado, R. y Quimbay, C. (2009). Interés de las empresas por la financiación de sus actividades de I+D+I: un análisis en el marco de las redes complejas para el sector industrial manufacturero colombiano. En J. Robledo, F. Malaver y M. Vargas (eds.), Encuestas, datos y descubrimiento de conocimiento sobre la innovación en Colombia (pp. 211-233). Bogotá: Fundación Cultural Javeriana de Artes Gráficas.

Rajan, R., and Zingales, L. (2003). Saving capitalism from the capitalists. New York: Crown Business. Rivera, J. y Ruiz, D. (2009). El desempeño financiero de las empresas innovadoras en Colombia. En: H. Rodríguez y V. Virgen (eds.), Memorias del encuentro de investigadores en prospectiva, innovación y gestión del conocimiento (pp. 128-144). Cali: Universidad del Valle.

Robb, A., and Robinson, D. (2014). The capital structure decisions of new firms. The Review of Financial Studies, 27 (1), 153-180.

Sierra, J., Malaver, F. y Vargas, M. (2009). La financiación de la innovación: un análisis a partir de la encuesta de innovación de Bogotá y Cundinamarca. En: J. Robledo, F. Malaver y M. 
Vargas (eds.), Encuestas, datos y descubrimiento de conocimiento sobre la innovación en Colombia (pp. 175-210). Bogotá: Fundación Cultural Javeriana de Artes Gráficas.

Soete, L.,Verspagen, B., and Weel, B. (2010). Systems of Innovation. In: B. Hall and N. Rosenberg (eds.), Handbooks of the economics of innovation (pp.1160-1180). Amsterdam: Elsevier.

Ughetto, E. (2008). The financing of innovative activities by banking institutions: policy issues and regulatory options. In: B. Laperche and D. Uzunidis (eds.), Powerful finance and innovations trends in a high-risk economy (pp. 224-247). London: Palgrave MacMillan.

University of Strathclyde (s.f.). Goodness of fit measures in logistic regression. Humanities y Social Sciences. Recuperado de: http://www.strath.ac.uk/aer/materials/5furtherquantitativeresearchdesignandanalysis/unit6/goodnessoffitmeasures/

Williamson, 0. (1988). Corporate finance and corporate governance. Journal of Finance, 43, 567-591.

Williamson, 0. (1996). The mechanisms of governance. Oxford, UK: Oxford University Press. Wooldridge, J. (2002). Econometric analysis of cross section and panel data. Cambridge, Massachusetts: The MIT Press.

Yin, R. (2003). Case Study Research, Design and Methods. (3rd. ed.). Newbury Park, California: Sage Publications.

\section{Tabla A1}

Definición de las variables independientes empleadas en los estudios de regresión múltiple

\begin{tabular}{|c|c|c|}
\hline Relacionadas con las fuentes de financiación: & $\begin{array}{c}\text { Media aritmética } \\
\%\end{array}$ & Descripción \\
\hline \multicolumn{3}{|c|}{ Financiación de Capital de Trabajo: Promedio de \% de Capital de Trabajo financiado por: } \\
\hline Fuentes internas/utilidades retenidas & 37,45 & \multirow{5}{*}{$\begin{array}{l}\text { Variables continuas que indican el \% de la financia- } \\
\text { ción total del Capital de Trabajo representado por la } \\
\text { respectiva fuente. }\end{array}$} \\
\hline Bancos & 21,15 & \\
\hline Instituciones financieras no bancarias & 1,48 & \\
\hline Crédito /avances proveed/clientes & 35,25 & \\
\hline Otros & 4,32 & \\
\hline \multicolumn{3}{|c|}{ Financiación de Activos Fijos: Promedio de \% de los Activos Fijos financiados por: } \\
\hline Fuentes internas /utilidades retenidas & 43,6 & \multirow{6}{*}{$\begin{array}{l}\text { Variables continuas que indican el \% de la financia- } \\
\text { ción total de los Activos Fijos representado por la } \\
\text { respectiva fuente. }\end{array}$} \\
\hline $\begin{array}{l}\text { Contribución de los dueños o emisión de } \\
\text { nuevas acciones }\end{array}$ & 4,17 & \\
\hline Bancos & 32,82 & \\
\hline $\begin{array}{l}\text { Préstamos de instituciones financieras no } \\
\text { bancarias }\end{array}$ & 4,39 & \\
\hline Crédito proveedores /Avance clientes & 11,9 & \\
\hline Otros & 3,09 & \\
\hline
\end{tabular}




\begin{tabular}{|c|c|c|}
\hline Relacionadas con las fuentes de financiación: & $\begin{array}{c}\text { Media aritmética } \\
\%\end{array}$ & Descripción \\
\hline \multicolumn{3}{|l|}{ Otras variables independientes } \\
\hline Edad & 23,41 & Variable que indica la edad de la firma en años. \\
\hline Tamaño & 2,04 & $\begin{array}{l}\text { Variable categórica según número de empleados: } \\
\begin{array}{l}1=\text { pequeña }(>=5<=19) ; 2=\text { mediana }(>=20<=99) \text { y } \\
3=\text { grande }(>=100) .\end{array}\end{array}$ \\
\hline Sector & 0,74 & $\begin{array}{l}\text { Variable ficticia }(0 / 1): 1 \text { si es del sector manufactu- } \\
\text { rero; }=0 \text { en cualquier otro caso. }\end{array}$ \\
\hline Estatus legal & 0,88 & $\begin{array}{l}\text { Variable ficticia (0/1): } 1 \text { si es una sociedad de res- } \\
\text { ponsabilidad Ltda. (Incluye listadas en mercados } \\
\text { públicos, de responsabilidad Ltda. no inscrita en } \\
\text { mercados públicos y sociedad de personas Ltda. y } \\
\text { de naturaleza privada); } 0 \text { en cualquier otro caso. }\end{array}$ \\
\hline Índice agregado de innovación & 1,876 & $\begin{array}{l}\text { Su descripción se muestra en } 3 \text {. Resultados y en la } \\
\text { Tabla } 6 .\end{array}$ \\
\hline Innovación como obstáculo & & $\begin{array}{l}\text { Variable ficticia }(0 / 1)=0 \text { si respondió negativamen- } \\
\text { te, y } 1 \text { en caso contrario. }\end{array}$ \\
\hline Cuenta bancaria & 0,99 & $\begin{array}{l}\text { Variable ficticia }(0 / 1)=1 \text { si tiene cuenta bancaria; } \\
=0 \text { en cualquier otro caso. }\end{array}$ \\
\hline Con inversión extranjera & 0,10 & $\begin{array}{l}\text { Variable ficticia }(0 / 1)=1 \mathrm{si} \% \text { poseído por individuos/ } \\
\text { compañías privadas extranjeras es diferente de cero; } \\
=0 \text { en cualquier otro caso. }\end{array}$ \\
\hline Estados Financieros Auditados & 0,62 & $\begin{array}{l}\text { Variable ficticia }(0 / 1)=1 \text { si tiene estados financieros } \\
\text { auditados; }=0 \text { en cualquier otro caso. }\end{array}$ \\
\hline Patentes & 0,22 & $\begin{array}{l}\text { Variable ficticia }(0 / 1)=1 \text { si tiene patentes registra- } \\
\text { das; }=0 \text { en cualquier otro caso. }\end{array}$ \\
\hline
\end{tabular}

Fuente: elaboración propia, con base en Enterprise Survey-Raw Data (2010). 
